\title{
Quantifying Profile Stiffness
}

\author{
Boudewijn Ph. VAN MILLIGEN, Raúl SÁNCHEZ1), Victor TRIBALDOS and Victor I. VARGAS \\ Asociación EURATOM-CIEMAT para Fusión, Avda. Complutense 22, 28040 Madrid, Spain \\ ${ }^{1)}$ Fusion Energy Division, Oak Ridge National Laboratory, P.O. Box 2001, Oak Ridge, TN 37831-2001, USA
}

(Received 13 November 2007 / Accepted 29 January 2008)

\begin{abstract}
Profile stiffness is quantified using a simple technique. The approach is tested on a paradigmatic numerical stiff transport model for one field (particles). The stiffness is found to exhibit radial structure and to depend on collisionality, which might help explaining the observed lack of stiffness in stellarators, as compared to tokamaks. The extension of this approach to heat transport requires some care. A proposal for a stiffness quantifier for heat transport is presented, and tested on data from the TJ-II stellarator.
\end{abstract}

(c) 2008 The Japan Society of Plasma Science and Nuclear Fusion Research

Keywords: profile stiffness, anomalous transport, critical gradient, tokamak-stellarator comparison

DOI: $10.1585 /$ pfr.3.S1070

\section{Introduction}

Profile stiffness (also known as profile consistency or resilience) is the striking phenomenon that temperature or pressure profiles tend to adopt the same shape, regardless of the applied drive, at least in a certain parameter range. The phenomenon is well established for tokamaks, but rarely found in stellarators [1]. This is slightly enigmatic, since power degradation is a universal phenomenon in stellarators, with a power dependence similar to that in tokamaks [2], and it is believed that threshold-triggered instabilities (leading to enhanced transport) should be operative both in tokamaks and stellarators. This leads to the expectation that profile stiffness should also be present in stellarators, if only to a less degree (and less obviously) than in tokamaks.

The detection of profile stiffness based on the direct comparison of profiles does not allow a quantification of the degree of stiffness, while the full 1-D modeling of transport requires making assumptions not related to the stiffness issue. Therefore, a stiffness quantifier is needed to resolve this issue. In the present work, we apply a standard quantifier for profile stiffness to a paradigmatic stiff particle transport model. We then discuss the possible (non-standard) extension of the method to heat transport and present first results for the TJ-II stellarator.

Profile stiffness can be understood as a sublinear response of profile amplitudes to a (small) change in drive. Pure diffusive transport models with fixed parameters produce a proportional response in profiles to changes in fuelling or heating, since the diffusion equation is linear in the profile amplitude and the source strength. Thus, the search for profile stiffness is closely related to the study of the dependence of transport coefficients on (gradients of) the transported quantity, since such a dependence would break the linearity of the diffusion equation.
Previous attempts to quantify profile stiffness (of the electron temperature profile) can be classified into two main phases. First, the work by Lopes Cardozo led to the introduction of the ratio of the incremental heat diffusivity (from perturbative analysis) to the steady state (power balance) value $\left(\chi^{\text {inc }} / \chi^{\mathrm{PB}}\right)[3,4]$. Although this important work has added significantly to the general understanding of the issue, this ratio of diffusivities does not provide an optimal quantifier in the sense that it is not tuned to the instability mechanisms involved (see the discussion below). Second and more recently, profile stiffness has been quantified on the basis of a phenomenological critical gradient model [5-10]. The main advance provided by this approach is the use of $\nabla T / T$ (the normalized temperature gradient) as a critical parameter, motivated by the theory of electron or ion temperature gradient (ETG/ITG) driven turbulence and the theory of drift trapped electron modes (DTEM), and in this respect, it has proved reasonably successful. However, a drawback of the analysis proposed in the cited references is that it requires postulating a specific phenomenological transport model, for which only partial justification can be provided. Our view is that stiffness is, in a sense, a "material property," so that it should be possible to quantify it independently from the transport model chosen, and consequently, we will define modelindependent (and therefore, bias-free) stiffness measures. This should allow distinguishing between the various instability mechanisms generating the observed profile stiffness.

\section{Numerical Study}

In the following, we will be studying transport using a simplified transport model that has been developed for particles alone. Thus, we will address the stiffness issue in the framework of particle transport first, leaving the discussion of heat transport, which is largely analogous, for 
later. Furthermore, while in fusion plasmas particle transport is generally considered not to be stiff, an anomalous particle pinch is commonly observed that might, in part, be due to nonlinearities of the transport equation, and which could perhaps be understood in the framework of the ideas expounded here. Following Ref. [4], one distinguishes between the steady state (or "power balance") transport coefficient $D^{\mathrm{pb}}=-\Gamma / \nabla n$ (here, $\Gamma$ is the particle flux and $\nabla n$ the density gradient) and the perturbation response value $D^{\text {inc }}=-\partial \Gamma / \partial \nabla n$, dubbed the "incremental" transport coefficient. If $D^{\text {inc }}>D^{\text {pb }}$, the profiles will respond sublinearly to changes in the source term, thus producing stiffness. Accordingly, a "stiffness factor" can been defined (by analogy to [6]):

$$
C=\frac{D^{\mathrm{inc}}}{D^{\mathrm{pb}}}=\frac{\nabla n}{\Gamma} \frac{\partial \Gamma}{\partial \nabla n},
$$

so that $C>1$ would indicate some degree of stiffness. While this analysis is completely conventional, we stress that it works only because the critical parameter of the model, which we will be examining in the following section, is designed to be critical with respect to $\nabla n$. We will return to this important point below.

The evaluation of $D^{\text {inc }}$ requires a (small) perturbation of the source term and profiles, either spontaneous or induced externally. However, the relevant variables of systems near a critical steady state tend to fluctuate spontaneously around a mean value. This property can be exploited to obtain another, equivalent estimate of the stiffness that does not require perturbing the system. Interpreting the mean amplitude of the fluctuations around the steady state values (i.e., their standard deviation) as the small change symbolized by $\partial$ in Eq. (1) [11]:

$$
D^{\text {fluct }}=\operatorname{RMS}(\Gamma) / \operatorname{RMS}(\nabla n)
$$

where $\operatorname{RMS}(f)=\left\langle(f-\langle f\rangle)^{2}\right\rangle^{1 / 2}$, and the angular brackets refer to a time average. In steady state, and assuming that the system response to perturbations is linear in the first approximation, we expect $D^{\text {fluct }} \simeq D^{\text {inc }}$.

In the following, we study the stiffness parameter $C$ using a simplified transport model, considered paradigmatic for transport controlled by a critical gradient. It is a simplified one-dimensional model for the transport of a conserved field (such as particles) in fusion plasmas, based on the continuous time random walk (CTRW) formalism [12,13], which provides a description of diffusive transport processes at a very fundamental level, and which leads to the generalized master equation (GME). Notably, this approach avoids making assumptions about locality of the transport processes, and allows critical gradient mechanisms to be treated in a mathematically sound manner. When incorporating a critical gradient mechanism, this model was shown to reproduce much of the unusual phenomenology observed in actual fusion experiments: power degradation, profile stiffness, rapid propagation of perturbations, "uphill" transport, and a transition of system size scaling reminiscent of the Bohm/gyro-Bohm scaling transition [14-19]. Taken together, these features convert this model into a somewhat "paradigmatic" model for stiff transport in fusion plasmas.

The simplified model is Markovian in nature and the time evolution of the single field $n(x, t)$, which may be interpreted as a (particle) density, obeys the following GME:

$$
\begin{aligned}
& \frac{\partial n(x, t)}{\partial t}=S(x, t) \\
& \quad+\frac{1}{\tau_{\mathrm{D}}} \int_{0}^{1} \mathrm{~d} x^{\prime} p\left(x-x^{\prime} ; x^{\prime}, t\right) n\left(x^{\prime}, t\right)-\frac{n(x, t)}{\tau_{\mathrm{D}}} .
\end{aligned}
$$

The domain of the system is $0 \leq x \leq 1$, implying a normalization of the spatial scales of the system to the system size. $\tau_{\mathrm{D}}$ is the waiting time and specifies the mean time a particle remains at a given location before taking a step. We set $\tau_{\mathrm{D}}=1$, implying a normalization of the time scales of the system to the mean waiting time. $S(x, t)$ is an external particle source and compensates edge losses due to the absorbing boundary conditions imposed at $x=0,1$. The function $p$ is a "particle step distribution." When $p$ is Gaussian, $p\left(x-x^{\prime}, x^{\prime}, t\right)=\exp \left[-\left(x-x^{\prime}\right)^{2} / 4 \sigma^{2}\right] / 2 \sigma \sqrt{\pi}$, standard diffusion is recovered in the limit of small step sizes $\sigma$ (and assuming a smooth density profile [18]):

$$
\frac{\partial n}{\partial t}=\frac{\partial^{2}}{\partial x^{2}}\left[\frac{\sigma^{2}}{\tau_{\mathrm{D}}} n\right]+S .
$$

Thus, the model is closely related to standard transport models.

The step distribution $p$ is chosen as follows to produce the required critical gradient mechanism:

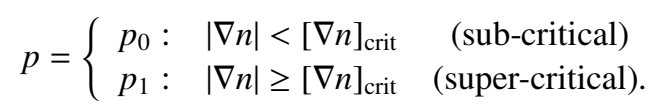

When the local gradient is below the critical value (subcritical), transport is governed by the $p_{0}$ step distribution, and when it is above (supercritical), it is governed by the $p_{1}$ step distribution. Here, $p_{0}$ and $p_{1}$ are fixed and symmetric stable probability distributions (of the Lévy type, of which the Gaussian is a special case). Transport at any given location $x$ will therefore be sub or supercritical as a function of the local value of the density gradient. This introduces a mechanism for self-regulation into the model.

In this work, $p_{0}$ is always chosen to be a Gaussian (with width $\sigma_{0}$ ), while $p_{1}$ can either be a Gaussian (with width $\left.\sigma_{1}\right)$ or a Cauchy distribution $p_{1}\left(x-x^{\prime}, x^{\prime}, t\right)=$ $\sigma_{1} / \pi\left(\sigma_{1}^{2}+\left(x-x^{\prime}\right)^{2}\right)$. While a Gaussian distribution models "normal" diffusive transport, a Cauchy distribution (with a "long tail") is used to model processes with long-range correlations, typically called "avalanches" or "streamers" in the plasma transport context, and representative of turbulent or "anomalous" transport.

To compute $D^{\text {inc }}$, we will be comparing steady state profiles at slightly different values of the amplitude of the 
source $S$. For simplicity, the steady state flux $\Gamma$ is computed as $\Gamma(x)=\int_{0.5}^{x} \mathrm{~d} x^{\prime} S\left(x^{\prime}\right)$. The lower limit of the integral corresponds to the system center at $x=0.5$. This calculation is sufficiently accurate for evaluating $D^{\text {inc }}$. However, for the calculation of $D^{\text {fluct }}$, we will use a different estimate of the flux that includes fluctuating contributions (see below).

The numerical calculations are performed in the domain $0 \leq x \leq 1$, on a grid with either $N=2000$ (high resolution) or $N=200$ (low resolution) grid points, using standard integration techniques for stiff differential equations to advance Eq. (3) in time. In all cases, the source $S(x, t)=S_{0}$ is considered constant. We first performed a scan of the source rate at high resolution (13 cases), and after checking that the results at lower resolution were equivalent, we performed a bi-dimensional parameter scan of both the source rate and the subcritical diffusion coefficient, the latter being proportional to $\sigma_{0}^{2}(130$ cases).

\subsection{Source scan}

More details on the high-resolution calculations discussed here can be found in Ref. [20]. We set $\sigma_{0}=0.002$, while $p_{1}$ is Gaussian with $\sigma_{1}=0.008$ for the cases labeled Gauss-Gauss or "GG" (both transport channels are Gaussian), or $p_{1}$ is Cauchy with $\sigma_{1}=0.004$ for the cases labeled Gauss-Cauchy or "GC." The critical gradient is chosen as $[\nabla n]_{\text {crit }}=2000$.

For both series (GG and GC), a scan of the source rate was performed, choosing $S_{0} \in\{0.01,0.02,0.05,0.1,0.2$, $0.5,10\}$. The parameter $D^{\text {inc }}$ was computed by comparing the profiles corresponding to two subsequent values of $S_{0}$.

Steady state profiles are shown in Fig. 1 and the corresponding gradients in Fig. 2. The GG and GC profiles are very similar, except for the highest fuelling rate $\left(S_{0}=0.5\right)$ : whereas the GC profile remains critical across the system (as is evident from the gradient, which remains below the critical value across most of the system), the GG profile "bulges," i.e., becomes supercritical. This difference can be ascribed to the larger transport capacity of the supercritical transport channel in the GC case.

In order to quantify the stiffness, we computed the stiffness factor $C$. Fig. 3 shows the radial profiles of the stiffness factor $C$ for the GG and GC cases. The spikes in the figure occur when the local values of the gradient $\nabla n(x)$ corresponding to the two subsequent values of $S_{0}$ being analyzed are equal. Such points should be ignored, since their statistical error is large, and thus, we will base the analysis on the global traces while ignoring the spikes. The figure shows that the critical region (where $C \gg 1$ ) grows inward from the edge as the source is increased. Next, a super critical region (with $C \simeq 1$ ) starts to grow inward from the edge. The supercritical state covers almost the entire system in the GG case at the highest fuelling rate, while in the GC case, it only affects a narrow boundary layer for the highest fuelling rate.

Fig. 4 shows $\langle C\rangle$, the radial average of $C$. The points

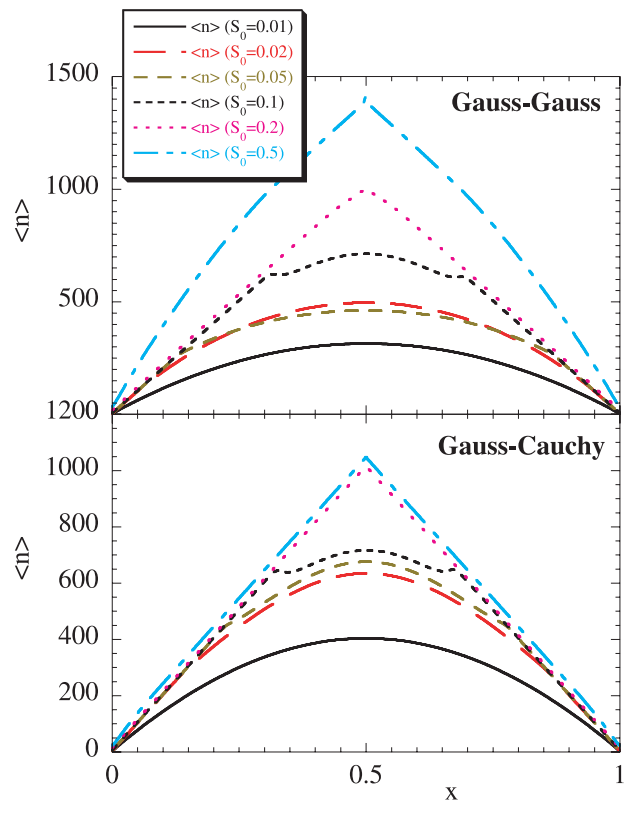

Fig. 1 Profiles vs. $S_{0}$ : Gauss-Gauss (top) and Gauss-Cauchy (bottom).

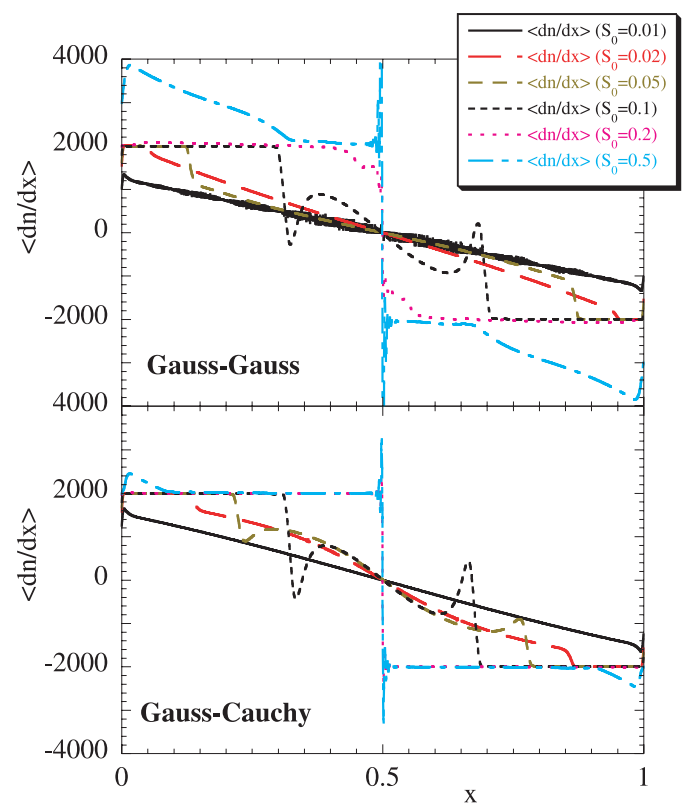

Fig. 2 Profile gradients vs. $S_{0}$ : Gauss-Gauss (top) and GaussCauchy (bottom).

in the figure are labeled by the lower of the two $S_{0}$ values used. In comparison to the GG cases, the GC cases do not only yield (slightly) larger values of $C$, but the range of values of $S_{0}$ where $C \gg 1$ exceeds the corresponding range for the $\mathrm{GG}$ cases.

\subsection{Bi-dimensional parameter scan (source rate and diffusivity)}

Here we scan the source amplitude $S_{0}$ and $\sigma_{0}$. The latter can be interpreted as a scan of the subcritical diffusivity (or "collisionality"). As in Ref. [14], we set $\tau_{\mathrm{D}}=1$, $S(x)=S_{0}$, and $[\partial n / \partial x]_{\text {crit }}=50$. To compute $C$, the same 


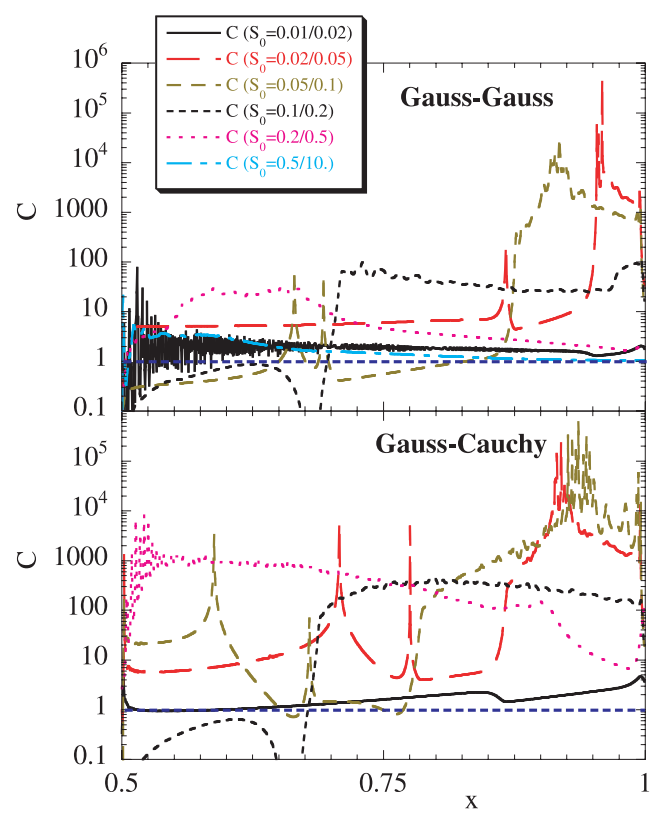

Fig. 3 Profiles of $C$ for different combinations of fuelling $S_{0}$.

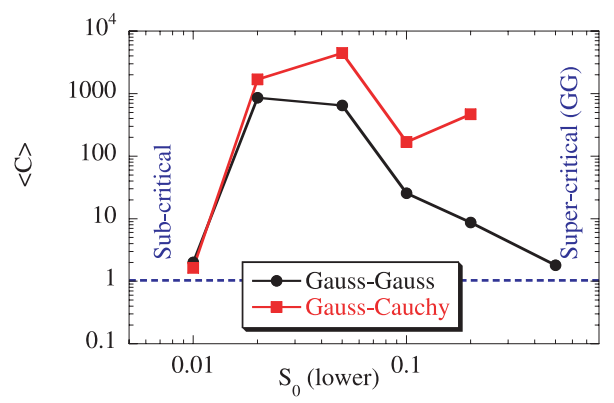

Fig. 4 Source scan, $\langle C\rangle$ vs. $S_{0}$.

runs were repeated with $S(x)=1.1 \cdot S_{0}$.

Fig. 5 shows $\langle C\rangle$ for the GG cases with $\sigma_{1}=0.08$, and for GC with $\sigma_{1}=0.04$. For GG, the largest possible value of $\sigma_{0}$ is $\sigma_{1}$. For GC, no such limit exists on $\sigma_{0}$. It is observed that $\langle C\rangle$ is a sensitive diagnostic parameter for criticality.

In all series studied, $\langle C\rangle$ is seen to increase gradually with increasing $\sigma_{0}$, reach a maximum value, and then drop somewhat abruptly and make a sharp transition to its subcritical expectation value $(C=1)$ at a precise value of $\sigma_{0}$. For the GC cases, the point where the system transits from a fully subcritical state to a critical state has been computed in a previous work [14]. This critical power threshold is given by $S_{\mathrm{c}}=2 \sigma_{0}^{2} / \tau_{\mathrm{D}}[\nabla n]_{\text {crit }}$ or $\sigma_{0}=\sqrt{S_{\mathrm{c}}} / 10$ with our choice of parameters. This matches the results exactly. For the GG cases, the transition point is set by the lesser of the cited threshold and the condition $\sigma_{0} \leq \sigma_{1}$ ( $\sigma_{1}$ cannot be smaller than $\sigma_{0}$, because the supercritical transport channel must provide faster transport than the subcritical transport channel for the critical mechanism to work).

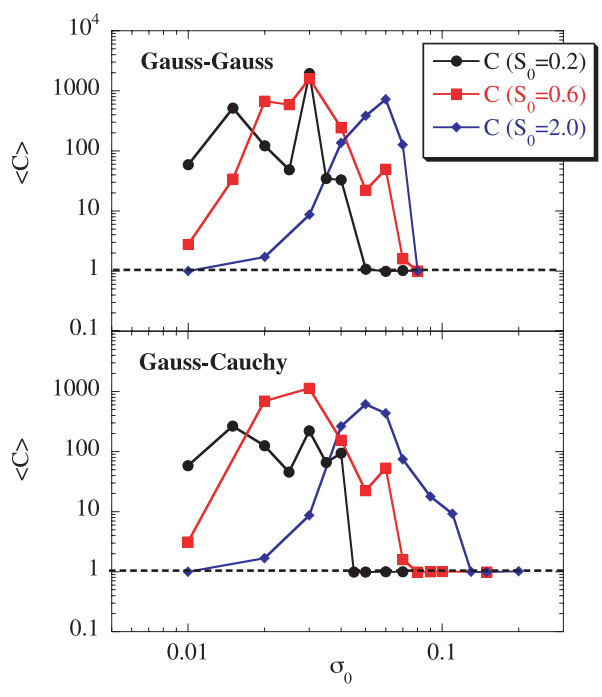

Fig. $5\langle C\rangle$ vs. $\sigma_{0}$ for various values of $S_{0}$ : Gauss-Gauss (top) and Gauss-Cauchy (bottom).

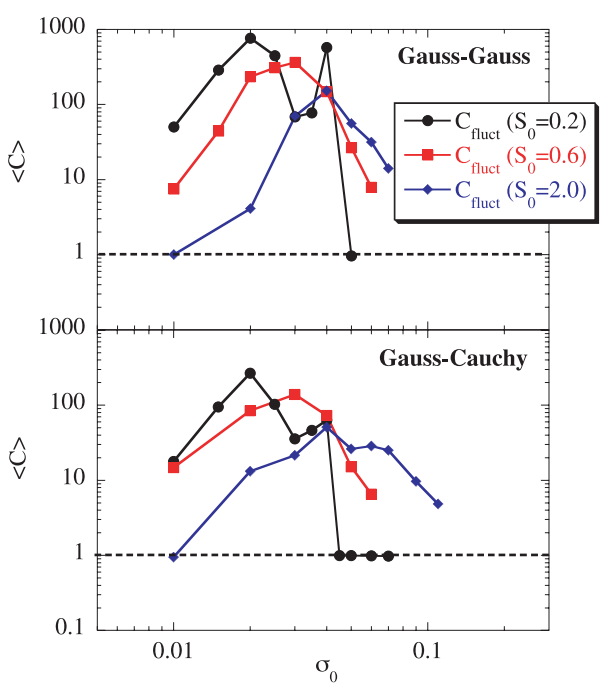

Fig. $6\left\langle C^{\text {fluct }}\right\rangle$ vs. $\sigma_{0}$ and $S_{0}$ : Gauss-Gauss (top) and GaussCauchy (bottom).

\subsection{Stiffness from fluctuations}

Above we also introduced a definition of stiffness from fluctuations (cf. Eq. (2)). In this section, we will evaluate this quantity for the cases studied here. The advantage of this stiffness measure over the previous one is that it does not require comparing the profiles of two separate steady states; however, it requires access to the fluctuating quantities. In the case of our numerical model, it is straightforward to compute the fluctuating gradient, while the flux can be evaluated from particle conservation $(\partial n / \partial t=-\partial \Gamma / \partial x+S)$ in combination with Eq. (3). Thus,

$$
\Gamma(x, t)=\int_{0}^{1} \mathrm{~d} x^{\prime} K\left(x-x^{\prime}, x^{\prime}, t\right) \frac{n\left(x^{\prime}, t\right)}{\tau_{\mathrm{D}}\left(x^{\prime}\right)},
$$

where $K\left(x-x^{\prime}, x^{\prime}, t\right)=\Theta\left(x-x^{\prime}\right)-P\left(x-x^{\prime}, x^{\prime}, t\right), \Theta(x)$ is the Heaviside function, and $P\left(\Delta, x^{\prime}, t\right)=\int_{-\infty}^{\Delta} \mathrm{d} x p\left(x, x^{\prime}, t\right)$ is the cumulative step probability distribution. 
Fig. 6 shows the calculation of $\left\langle C^{\text {fluct }}\right\rangle$. Compare these results to Fig. 5. Although the maximum numerical value of $\left\langle C^{\text {fluct }}\right\rangle$ is somewhat lower than that of $\langle C\rangle$, the global trend is the same. The deviation between $C$ and $C^{\text {fluct }}$ at points with large stiffness is to be expected, as the system response will be strongly nonlinear at such points. The calculation of $C^{\text {fluct }}$ is not possible when the system is locally static, which explains why these figures have less data points than Fig. 5.

\section{Extension to Heat Transport}

The preceding analysis was simplified by the fact that the thermodynamic force and the critical parameter were both equal to $\nabla n$. For heat transport, these two quantities do not coincide, and the definition of a stiffness quantifier is less obvious. The traditional proposal, $C=\chi^{\mathrm{inc}} / \chi^{\mathrm{pb}}=$ $-\partial q / \partial(n \nabla T) / \chi[6]$, where $\chi$ is the heat diffusivity, will respond only weakly when the system criticality is not determined by $n \nabla T \simeq$ constant. In general, a stiffness quantifier $C$ that responds sharply to a given critical condition should be proportional to the inverse of the change in that critical condition, as might indeed be deduced from the modeling efforts in, e.g., [5]. Since it is being suggested that ITG/ETG/DTEM might play a role in the stiffness (if any) of the temperature profile, a suitable choice for the critical parameter might be $\nabla T / T$ [21]: therefore, we define the stiffness of the temperature profile by

$$
C^{\mathrm{L} T \mathrm{e}}=\frac{1}{\chi} \frac{\partial(\chi \nabla \ln T)}{\partial(\nabla \ln T)} .
$$

This unorthodox proposal is designed to detect the dependence of the heat diffusivity, $\chi$, on the expected critical parameter for the ETG instability, $\nabla T / T$. We note that many alternative definitions are possible. In fact, one might devise a specific definition for every threshold-triggered instability suspected to be operative in fusion plasmas. Perhaps such an approach can be used to identify the different instabilities involved in regulating the profiles.

\section{Application to the TJ-II Stellarator}

Here, we report the first attempt to estimate the stiffness of the temperature profile in the stellarator TJ-II. Profiles at TJ-II are obtained by single-pulse high-resolution Thomson Scattering diagnostic [22], yielding around 200 data points for the electron temperature $T$ and density $n$ along a chord spanning most of the plasma cross section, with a spatial resolution of $2.25 \mathrm{~mm}$.

The goal of the present analysis is to determine the global transport response to a change in heating. Therefore, we fit the profiles to simple functional forms, thus ignoring any detailed radial structure. This improves the robustness of the calculation of radial derivatives necessary to compute $C^{\mathrm{L} T \mathrm{e}}$. The temperature profile is fitted to the sum of two Gaussians, while the density profile is fitted to a Gaussian multiplied by a second-order polynomial in

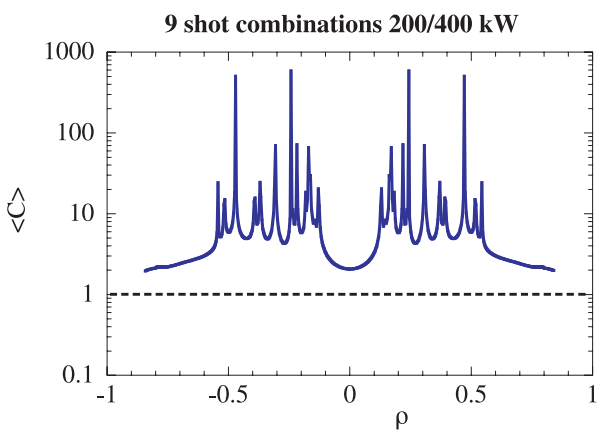

Fig. 7 Stiffness estimate for TJ-II.

$\rho^{2}$ (for symmetry). $\rho=\sqrt{\psi}$ is a radial coordinate, where $\psi$ is the normalized poloidal magnetic flux, obtained from the theoretical calculation of the magnetic flux surfaces in vacuum. Finite pressure effects can be safely ignored. The discharges studied here are those reported in Ref. [23].

The error in the profile reconstruction, evaluated using the Jacobian of the fit matrix, is of the order of $10 \%$, lower at the center but increasing towards the edge. While the temperature profile reconstruction is reliable (i.e., with an error less than $10 \%$ ) to about $\rho=0.7$, the density profile reconstruction is reliable only to about $\rho=0.4$. The calculation of $C^{\mathrm{L} T \mathrm{e}}$ is not very sensitive to the details of the density profile.

To compute $\chi$ and $C^{\mathrm{L} T \mathrm{e}}$, an estimate of the heat flux $q$ is required. The heat source is assumed to have a Gaussian deposition profile, centered at $\rho=0$, with a fixed width of $\Delta \rho=0.2$. The heating efficiency is estimated to be $60 \%$. Radiation and other losses are ignored. The heat flux is obtained by integrating the net deposited power. In any case, the stiffness factor $C^{\mathrm{L} T \mathrm{e}}$ is not very sensitive to the details of this calculation. Fig. 7 shows the mean stiffness factor, averaged over 9 equivalent discharge combinations with similar densities and different heating levels. The spikes in the curves of $\left\langle C^{\mathrm{LTe}}\right\rangle$ are not significant, as they correspond to radial points where the curves of $\nabla \ln T$, corresponding to the two discharges being compared, cross. At such points, both the value and error of $C^{\mathrm{L} T \mathrm{e}}$ are infinite. However, ignoring the spikes, one observes that a certain profile stiffness exists $\left(\left\langle C^{\mathrm{L} T \mathrm{e}}\right\rangle>1\right)$ in the radial range $0.15 \leq \rho \leq 0.55$, roughly coincident with the $T$ gradient region.

\section{Discussion}

The quantification of profile stiffness is directly related to the detection of the dependence of the transport coefficient on the profile gradient. In accordance with this idea and with literature, we use a stiffness quantifier $C$, and show that it provides a useful quantification of stiffness in a paradigmatic transport model. It appears that stiffness has a radial structure and depends on system parameters (such as the source or drive and the collisionality), which could possibly explain the observed differences between 
tokamaks and stellarators.

The extension of these results to heat transport requires some care. In this work, we suggest that the definition of stiffness can be tuned to the critical mechanism suspected to be regulating the profiles. This tuning then means that the stiffness quantifier responds sharply when the suspected mechanism is indeed operative. More than one mechanism can be contemplated, and corresponding stiffness quantifiers can be defined. Thus, it may be possible to establish the parameter ranges and locations where each mechanism operates.

Finally, we have applied a particular stiffness quantifier to TJ-II data, and obtained a moderate degree of stiffness.

\section{Acknowledgment}

This research was sponsored in part by Dirección General de Investigaciones Científicas y Tecnológicas (DGICYT) of Spain under Project No. ENE2004-04319. Part of this research was sponsored by the Laboratory Research and Development Program of the Oak Ridge National Laboratory, managed by UT-Battelle, LLC, for the US-DOE under contract number DE-AC05-00OR22725.

[1] U. Stroth, Plasma Phys. Control. Fusion 40, 9 (1998).

[2] B. Carreras, IEEE Trans. Plasma Sci. 25, 1281 (1997).

[3] N. Lopes Cardozo, J. de Haas, G. Hogeweij, J. O'Rourke, A. Sips and B. Tubbing, Plasma Phys. Control. Fusion 32, 983 (1990)

[4] N. Lopes Cardozo, Plasma Phys. Control. Fusion 37, 799 (1995).

[5] F. Imbeaux, F. Ryter and X. Garbet, Plasma Phys. Control. Fusion 43, 1503 (2001).

[6] F. Ryter, F. Leuterer, G. Pereverzev, H.-U. Fahrbach, J. Stober, W. Suttrop and ASDEX Upgrade Team, Phys. Rev.
Lett. 86, 2325 (2001).

[7] X. Garbet, P. Mantica, F. Ryter, G. Cordey, F. Imbeaux, C. Sozzi, A. Manini, E. Asp, V. Parail, R. Wolf et al., Plasma Phys. Control. Fusion 46, 1351 (2004).

[8] X. Garbet, P. Mantica, C. Angioni, E. Asp, Y. Baranov, C. Bourdelle, R. Budny, F. Crisanti, G. Cordey, L. Garzotti et al. Plasma Phys. Control. Fusion 46, B557 (2004).

[9] F. Ryter, Y. Camenen, J. DeBoo, F. Imbeaux, P. Mantica, G. Regnoli, C. Sozzi, U. Stroth and ASDEX Upgrade, DIIID, FTU, JET-EFDA contributors, TCV, Tore Supra and W7-AS Teams, Plasma Phys. Control. Fusion 48, B453 (2006).

[10] P. Mantica and F. Ryter, Comptes Rendus Physique 7, 634 (2006).

[11] B. Carreras, V. Lynch, P. Diamond and M. Medvedev, Phys. Plasmas 5, 1206 (1998).

[12] E. Montroll and G. Weiss, J. Math. Phys. 6, 167 (1965).

[13] R. Balescu, Phys. Rev. E 51, 4807 (1995).

[14] B. van Milligen, R. Sánchez and B. Carreras, Phys. Plasmas 11, 2272 (2004).

[15] B. van Milligen, B. Carreras and R. Sánchez, Phys. Plasmas 11, 3787 (2004).

[16] B. van Milligen, P. Bons, B. Carreras and R. Sánchez, Eur. J. Phys. 26, 913 (2005).

[17] R. Sánchez, B. Carreras and B. van Milligen, Phys. Rev. E 71, 011111 (2005).

[18] R. Sánchez, B. van Milligen and B. Carreras, Phys. Plasmas 12, 056105 (2005)

[19] J. Gavnholt, J. Rasmussen, O. Garcia, V. Naulin and A. Nielsen, Phys. Plasmas 12, 084501 (2005).

[20] B. van Milligen, B. Carreras, V. Lynch and R. Sánchez, Nucl. Fusion 47, 189 (2007).

[21] X. Garbet, N. Dubuit, E. Asp, Y. Sarazin, C. Bourdelle, P. Ghendrih and G. Hoang, Phys. Plasmas 12, 082511 (2005).

[22] C. Barth, F. Pijper, H. van der Meiden, J. Herranz and I. Pastor, Rev. Sci. Instrum. 70, 763 (1999).

[23] V. Vargas, D. López-Bruna, J. Herranz and F. Castejón, Nucl. Fusion 47, 1367 (2007). 